\title{
Valor y valoración: discusión con el emotivismo y el institucionalismo
}

Jacob Buganza

Universidad Veracruzana, México

(c) $\underset{\mathrm{Br}}{\mathrm{i}}$ 


\title{
Valor y valoración: discusión con el emotivismo y el institucionalismo*
}

Resumen: este trabajo tiene como propósito retomar un apunte que nos parece importante del gran filósofo de la lógica Bocheński. Su apunte nos permite discutir con algunas corrientes filosóficas contemporáneas, especialmente situándonos en el llamado cognitivismo u objetivismo moral. Con base, pues, en tal apunte, discutimos con Hume y Smith, a quienes evaluamos como emotivistas, así como con Searle y Ferber, a quienes vemos en el institucionalismo. El trabajo intenta poner de manifiesto algunos puntos en donde hay convergencias con el objetivismo moral, y discute algunos otros en donde encontramos desacuerdos importantes.

Palabras clave: valor, valoración, emotivismo, cognitivismo, institucionalismo.

\section{Value and Valuation: A Discussion with Emotivism and Institutionalism}

\begin{abstract}
The purpose of this work is to take up a note that seems important to us from the great philosopher of logic, Bocheński. His note allows us to discuss with some contemporary philosophical schools, by placing us in the so-called cognitivism or moral objectivism. Based on that note, then, we discussed with Hume and Smith, whom we evaluate as emotivists, as well as with Searle and Ferber, whom we see in institutionalism. The work tries to highlight some points where there are convergences with moral objectivism, and discusses some others where we find important disagreements.
\end{abstract}

Keywords: value, valuation, emotivism, cognitivism, institutionalism.

Fecha de recepción: 18 de noviembre de 2016 Fecha de aceptación: 26 de julio de 2017

Forma de citar (APA): Buganza, J. (2018). Valor y valoración: discusión con el emotivismo y el institucionalismo. Revista Filosofía UIS, 17(1), doi: http://dx.doi. org/10.18273/revfil.v17n1-2018010

Forma de citar (Harvard): Buganza, J. (2018). Valor y valoración: discusión con el emotivismo y el institucionalismo. Revista Filosofía UIS, 17(1), 205-224.

Jacob Buganza: italiano-mexicano. Doctor en Filosofía. Adscrito al Instituto de Filosofía de la Universidad Veracruzana.

Correo electrónico: jbuganza@uv.mx

* Artículo de reflexión derivado de investigación. 


\section{Valor y valoración: discusión con el emotivismo y el institucionalismo}

\section{Introducción}

En este trabajo, nos proponemos retomar un apunte que efectúa Bocheński y que nos parece imprescindible para caracterizar al valor. Se trata sólo de un apunte que tomaremos como pretexto para discutir con otras posturas morales a partir de lo que en la filosofía contemporánea suele considerarse cognitivismo, que en la mejor tradición clásica habría que denominar objetivismo moral. Teniendo presente la distinción entre valor y valoración, efectuaremos un análisis y discusión con las posturas de David Hume, Adam Smith, como representantes del emotivismo, y con John Searle y Rafael Ferber, como paradigmas del institucionalismo moral. Veremos algunos puntos en los que concordamos, y mostraremos nuestras propias tesis a la luz de las de ellos.

\section{El planteamiento de Bocheński}

El planteamiento sobre el problema del valor a partir del pensamiento de Józef Maria Bocheński puede rastrearse en su pequeño libro Wege zum philosophischen Denken. Einführung in die Grundbegriffe (traducido como Introducción al pensamiento filosófico). Ahí, el filósofo polaco dedica un apartado al tema del valor. Se trata de un capítulo breve, como la totalidad de los que conforman dicho libro, pero, al igual que los otros, sustancioso.

Bocheński parte de una evidencia que no es necesario corroborar, a saber, que el hombre no sólo concibe la realidad en el sentido de que hace $\theta \varepsilon \omega \rho i ́ \alpha$ sobre ella, sino que la realidad le afecta indefectiblemente. El hombre no sólo ve la realidad, sino que la valora o estima. Se trata de una constatación que han tomado como punto de partida varias corrientes filosóficas, como el aristotelismo y la escolástica que lo sigue más de cerca, así como otras concepciones como el rosminianismo y la ética fenomenológica. 
El hombre, que irremediablemente se enfrenta a la realidad, la estima prácticamente todo el tiempo, aunque lo hace en distintos grados. En este sentido, dice el filósofo polaco: "El hombre siente la realidad como bella o fea, como buena o mala, como agradable o penosa, como noble o vil, como santa o no santa" (Bocheński, 1992, p. 69). En efecto, el hombre no apetece la realidad en bloque, sino que la apetece de distintas maneras de acuerdo con distintas facultades $y$, por ello, clasifica sus apetencias en grupos, a los cuales suelen llamarse, a partir de Max Scheler, "jerarquías axiológicas". Sin embargo, no es a esto último a lo cual se refiere Bocheński en su trabajo, sino que se enfoca más bien a dilucidar la naturaleza del valor en vez de distinguir qué facultades entran en juego en la valoración. En otras palabras, Bocheński parte del supuesto de que hay facultades que permiten al hombre valorar las cosas, aunque no se preocupa de la pregunta de si es capaz de visualizarlas tal cual son y, en este sentido, estaría forzado a valorarlas de tal suerte. Pero retomemos el sendero del polaco.

Con respecto a la naturaleza del valor, Bocheński distingue tres ámbitos. En primer lugar, es necesario destacar que la cosa, esto es, lo real, es valioso. En segundo lugar, hay que entresacar que lo que hace valioso a algo es alguna propiedad, lo cual es propiamente lo que se llama "valor". Por último, hay que distinguir entre "nuestra intuición de los valores, nuestra voluntad que apetece o repele algo" (1992, p. 73). El primer ámbito corresponde al portador del valor, el segundo corresponde al valor mismo y el tercero pertenece a la "actitud humana" frente al valor.

Esta serie de distinciones resultan interesantes porque permiten llevar a cabo un análisis lógico de lo que esencialmente es un valor, el cual, como se aprecia, parece darse en el entrecruce que se establece entre un portador y la actitud humana. Siendo así, el puente entre ambos es, en definitiva, lo que solemos conocer como "valor". Prestando atención a estos apuntes, se aprecia que Bocheński, con esta triple distinción, brinda pistas importantes sobre su tesis de fondo, pues el valor resulta ser un ámbito aparte del portador y de la valoración. Empero, no es precisamente el tema antedicho sobre el cual se centra el filósofo polaco, sino más bien en el tercero de los tres elementos, o sea, en la actitud humana, que es sobre la cual nos interesa reflexionar en este apartado.

La actitud humana frente al valor suele llamarse "valoración". La ética tiene carta de ciudadanía aquí, pues, como dice Ferber en su Philosophische Grundbegriffe (Conceptos fundamentales de la filosofía), "cuando decimos lo que es bueno, lo expresamos en frases que no son únicamente de naturaleza descriptiva, sino también valorativa" (1995, p. 151). En este asunto nuevamente se parte de una constatación: el hombre individual, la comunidad y las distintas civilizaciones, han valorado las cosas. Pero también es cierto que a través del tiempo la valoración sufre mutaciones. La constatación individual es irrefutable: cada uno ha valorado de distintas maneras una misma cosa; con el tiempo y las nuevas circunstancias, la 
valoración tiende a cambiar. De acuerdo con Bocheński, hay dos grandes teorías filosóficas que se han planteado resolver esta constatación al responder por qué se da este cambio. La primera, denominada positivista, "representada sobre todo por los positivistas británicos, afirma que la relatividad y variación de los valores se explica por la relatividad y variación de los valores mismos" (Bocheński, 1992, p. 75). Por otra parte, se encuentran los idealistas: "Están de acuerdo con que nuestras estimaciones varían y muchas cosas que aquí se miran como buenas son vistas en otra parte como malas. Sin embargo, hacen notar que esto no sucede sólo en el orden los valores" (76).

Pero para los idealistas el panorama de los valores es análogo al orden geométrico o al matemático, en donde algunas fórmulas han ido adquiriendo mayor exactitud con el paso del tiempo desde la perspectiva subjetiva, pero no parece que objetivamente. Para el idealismo, los valores morales, al igual que las relaciones matemáticas y geométricas, no cambian en sí, sino que cambia la manera en que se perciben. Una cosa es, pues, la valoración, y otra el valor. Esta distinción tiene capital importancia para el tema de los valores, en general, y especialmente en relación al valor moral, como intentaremos destacar más abajo.

Contra los positivistas, Bocheński asegura que se trata de una postura que no es sostenible porque confunde la valoración con el valor. En cambio, el idealismo posee la ventaja de que no necesita negar la evidencia inmediata de los valores. Pero, a diferencia de los idealistas, en donde seguramente agrupa a Scheler, escribe Bocheński:
Yo no pondría los valores en ningún cielo platónico. Sólo tienen consistencia en nuestro espíritu, exactamente como las leyes matemáticas. En el mundo sólo hay cosas particulares y, desde luego, reales [...] Los valores tienen cierto fundamento en el mundo [...] Los valores están fundados en la relación entre el hombre y las cosas" (1992, p. 78).

Como se ve en esta última cita, el filósofo polaco busca una posición intermedia que dé su justo puesto a las cosas valiosas y a la valoración. Nos parece que una posición como la del filósofo polaco es destacable y adecuada, en cuanto toma en cuenta tanto al sujeto que valora como al objeto valorado, y en su entrecruce sitúa la valoración. Y es que, recordando lo que anotamos, sólo puede apreciarse humanamente el valor concibiéndolo como la valoración (que puede ser moralmente laudable o vituperable) que se efectúa sobre alguna cosa que posee, por derecho propio, ciertas propiedades consideradas objetivamente valiosas. Ésta es, pues, nuestra posición de fondo, y con ella queremos dialogar con otras posturas. 
Con esta última puntualización efectuada, intentemos profundizar un poco más en estas clasificaciones agregando al caso lo que ha venido a llamarse "institucionalismo". Para ello, retomemos algunas indicaciones que elabora Rafael Ferber en su libro ya mencionado. Este filósofo alemán insiste constantemente en la importancia del lenguaje. Concretamente, en este caso, es importante subrayar la importancia del lenguaje de la moral. Resulta evidente que el hombre efectúa valoraciones y que éstas las expresa a través del lenguaje. Pero el lenguaje de la moral es más que valoraciones, a saber, implica prohibiciones, permisiones, órdenes, etcétera. Las aseveraciones morales, de acuerdo con este autor, se explican de distintas maneras en el seno de la filosofía moral, lo cual da origen a diversas teorías éticas que intentan dar cuenta de aquéllas. Se trata del cognitivismo, el emotivismo y el institucionalismo. Para no perder este hilo conductor, revisemos qué es el cognitivismo, pues nos dará la pauta para hablar tanto del emotivismo como del institucionalismo.

\section{Cognivitismo y emotivismo}

El cognitivismo sostiene un objetivismo y realismo morales, pues considera que las afirmaciones morales tienen el mismo estatuto que las afirmaciones con las cuales se expresa cualquier otro conocimiento. Tales afirmaciones son susceptibles de ser verdaderas o falsas, si es que coinciden o no con los "hechos morales". Al interior de estos hechos morales, es posible distinguir entre unos fundamentales y otros derivados. Por ejemplo, la dignidad humana resultaría ser fundamental, mientras que el derecho a la vida es derivado, por la sencilla razón de que el derecho a la vida obtiene su validez gracias a que la vida humana es digna y, por tanto, ha de ser respetada. Ahora bien, es frecuente encontrar un contraargumento común contra esta postura, a saber, que las cualidades morales son inobservables; las cualidades morales no provienen de los sentidos externos. No parece que las cualidades morales como "bueno" o "malo" puedan sentirse de la misma manera que aquellas que denominamos "blanco" o "negro". Es aquí donde las distintas posturas filosóficas que comparten el objetivismo se separan. Y es también aquí donde Ferber no parece ser muy certero en su trabajo, pues reduce todas las posturas objetivistas al intuicionismo (1995, pp. 154-155). Resulta ser verdad que varias corrientes objetivistas contemporáneas se reducen al intuicionismo, en donde la guerra de intuiciones es imposible que conduzca a un acuerdo, pues no es posible que alguien brinde un mejor argumento que otro debido a que se trata de intuiciones, y la intuición, por definición, excluye el discurso.

Empero, la intuición moral no debe quedar descartada. Tiene una función substancial que es imposible dejar de lado. En efecto, en varias ocasiones la intuición de un valor es la que brinda al agente moral la pauta para efectuar su deliberación, su consecuente elección y su actuar moral externo. Pero no parece conveniente postular sólo una facultad intuitiva moral que dé cuenta del valor 
moral, pues entonces el diálogo quedaría cancelado. Y cancelado el diálogo, la deliberación sobre lo que debe hacerse o no, sobre lo bueno o malo, quedaría descartada. De ahí que sea conveniente, en vistas a una mejor comprensión del fenómeno moral, postular una facultad que implique a la intuición, pero que no se disuelva en ella. Esta facultad es capaz de intuir, pero también lo es de argumentar, esto es, de deliberar; de tal manera, esta facultad es capaz de dar cuenta de por qué es mejor un acto que otro, o por qué es bueno moralmente un acto y otro, en cambio, es malo.

Nosotros proponemos que esta facultad capaz de dar cuenta de por qué es bueno o malo un acto, y que es una facultad constituida por la intuición original del ser, es la inteligencia, la cual se mueve dando razones. La inteligencia, en cuanto entendimiento, es capaz de intuir lo que son las cosas, sin que esto implique un conocimiento absoluto de lo conocido, pero al menos sí de lo que esencialmente es la cosa. Esta tesis, enmarcada en la antropología filosófica, es la que permite dar cuenta de que el hombre sea susceptible de la moralidad, pues si al hombre estuviera impedido el conocimiento de lo que es la cosa, le estaría imposibilitada una necesidad moral para valorar justamente a la cosa. Si esta necesidad para valorar no se da en el hombre, entonces no hay moralidad alguna. Gracias a que el hombre es capaz de concebir lo que es la cosa, es capaz también de argumentar por qué una valoración es razonable o no, entendiendo por "racionalidad" efectivamente el conocimiento de lo que es la cosa, que brinda, por decirlo así, la regla próxima de moralidad que funge como sustento para argumentar que algo debe valorarse de una forma y no de otra.

Pero además del problema facultativo, el cognitivismo se enfrenta a la llamada "falacia naturalista" o "ley de Hume". En el célebre is-ought passage (paso del ser al deber-ser), Hume dice que es inconcebible que se dé el paso del ser o no ser al deber o no deber; "cómo es posible que esta nueva relación se deduzca de otras totalmente diferentes" (Hume, 1998, p. 469). Siendo así, se verá que "la distinción entre vicio y virtud, ni está basada meramente en relaciones de objetos, ni es percibida por la razón" (470). De acuerdo con la supuesta falacia de Hume, es "inimaginable por completo" pasar del ser al deber-ser, es decir, de la descripción a la perlocución. Hume es uno de los más preclaros exponentes y defensores del emotivismo, que reduce el problema moral a los siguientes términos: toda proposición moral sólo refleja un estado emotivo y no propiamente un conocimiento. Por eso es que Bocheński suele referirse a la confusión entre el valor y la valoración como una herencia británica. Pero veamos el argumento central de Hume al respecto: 
Dado que la moral influye en las acciones y afecciones, se sigue que no podrá derivarse de la razón, porque la sola razón no puede tener nunca una tal influencia, como ya hemos probado. La moral suscita las pasiones y produce o impide las acciones. Pero la razón es de suyo absolutamente impotente en este caso particular. Luego las reglas de moralidad no son conclusiones de nuestra razón (1998, p. 457).

Hume considera que nadie puede dudar de esta conclusión. Pero analicémosla parte por parte: (i) asegura que la moral influye en las acciones y afecciones; (ii) la razón no puede influir en las acciones y afecciones; (iii) la moral suscita las pasiones y produce o impide las acciones; (iv) las reglas de moralidad no son conclusiones de la razón, pues ésta no influye en la moral.

Con respecto a (i), donde asegura que la moral influye en las acciones, puede concederse, pues debido a que hay moralidad es que pueden darse acciones con su carácter de buenas o malas; en cuanto a las afecciones, hay que distinguir exactamente qué quiera decirse con influencia, pues si se refiere a que la moral suscita las afecciones, resulta del todo falso, dado que las afecciones son movimientos del apetito en orden a un objeto, como cuando un agente sensitivo es afectado por el miedo que le produce un objeto que puede dañarlo o destruirlo de algún modo. Pero si la influencia a la cual se refiere Hume es a que la moral es capaz no de suscitar, sino de moldear las afecciones, entonces resulta certera su tesis. Pero, debido a que lo que intenta es negar la influencia de la razón en la esfera moral, y siendo la moral dependiente de la racionalidad, luego lo que sostiene es que la moral suscita para él las afecciones; mas hemos visto que es falso; en consecuencia, ya la premisa del argumento del británico resulta errónea.

Con respecto a (ii), la tesis es parcialmente verdadera, a saber, en lo que se refiere a los afectos; pero es falsa en lo que se refiere a la acción. Si se tiene en cuenta que unas líneas antes de enunciar este razonamiento Hume recurre a la distinción entre philosophia theorica y philosophia practica, entonces no es posible aceptar la tesis de que la razón no influya en la acción, pues la acción es un acto voluntario del agente moral; y si la voluntad es el apetito racional, luego la razón sí tiene injerencia en la acción; en consecuencia, nuevamente se aprecia la falsedad del argumento humeano.

Con respecto a (iii), resulta parcialmente verdadera y falsa la tesis del escocés: la moral no suscita las pasiones o afectos, sino que se trata de algo natural al apetito, es decir, los afectos se generan por su propio movimiento, pues es natural, por ejemplo, sentir miedo ante el objeto dañino; por otro lado, es verdad que la moral suscita o impide las acciones, pues se trata de un acto racional (no como quiere Hume, ciertamente). La falsedad de (iv) es ya patente, pues la razón sí influye en la acción a través de la moralidad y, de manera remota, en el moldeo de los afectos. 
En el fondo, Hume confunde las facultades humanas, a saber, una tripleta de apetitos que acaecen en el hombre. Todo género de apetito es colocado por Hume en una sola facultad, lo cual vuelve imposible las distinciones que hemos realizado. Además, da la impresión de que el filósofo británico confunde constantemente afección con acción. Hume niega que la razón sea una facultad activa: la considera "inactiva". Limita arbitrariamente la razón a descubrir la verdad o la falsedad en las relaciones reales de ideas o con la existencia de ellas; así, "Todo lo que no sea susceptible de tal acuerdo o desacuerdo es incapaz de ser verdadero o falso, y en ningún caso puede ser objeto de nuestra razón" (Hume, 1998, p. 458). Aunque esta tesis resulta cuestionable, a partir de ella concluye:

Es evidente que nuestras pasiones, voliciones y acciones son incapaces de tal acuerdo o desacuerdo, en cuanto que son hechos y realidades originales completos en sí mismos, sin implicar referencia alguna a otras pasiones, voliciones y acciones. Es imposible, por consiguiente, que puedan ser considerados verdaderos o falsos, contrarios o conformes a la razón (458).

Al limitar la razón al acuerdo o desacuerdo expresado en la verdad y la falsedad, que en el fondo señala el acuerdo o desacuerdo de las ideas y las proposiciones, arguye que las pasiones, voliciones y acciones concuerdan o no con otras pasiones, voliciones y acciones. Luego, no son objeto de la razón. Pero como las potencias no se limitan a constatar dicho acuerdo, entonces no se sigue que no sea activa en un cierto modo. Es más, la razón sí es efectiva en relación a las voliciones y a las acciones, pues las primeras son una apetencia razonada y las segundas se refieren a los actos externos que lleva a cabo el agente moral, acordes o no, eso sí, con la apetencia razonada. De ahí que resulte falsa la tesis inmediata de Hume: "Las acciones pueden ser laudables o censurables, pero no razonables o irrazonables" (1998, p. 458). Al contrario, debido a que las acciones se vinculan estrechamente con las voliciones, y siendo las voliciones apetencias razonadas, entonces pueden ser éstas razonables o irrazonables; y siendo razonables o irrazonables, entonces pueden ser laudables o vituperables en relación con su razonabilidad o irracionabilidad.

Hume no estaría en consonancia con lo último. Para él, la razón es incapaz de hallar lo bueno o lo malo moral por ser incapaz de influir en las acciones. Las limitaciones impuestas a la razón por parte de Hume lo conducen a sostener tal tesis. Y es que, para él, el entendimiento sería capaz de determinar lo bueno y lo malo (esto es, lo virtuoso y lo vicioso) sólo en alguna relación de objetos o en algún descubrimiento del razonamiento (Hume, 1998, p. 463). Pero es precisamente la relación de un objeto con un sujeto aquello que hace a algo bueno o malo, y es lo que Hume no vislumbra, como sí lo hace, por cierto, Bocheński. Por ejemplo, el alcohol y el bebedor tienen una mala relación en cuanto el primero destruye al segundo. Esta relación, fincada en los hechos, es la que pone de manifiesto la maldad que reporta para el bebedor el alcohol. El problema que se aprecia en 
la visión sobre la razón que Hume sostiene es que considera que ésta procede de manera geométrica necesariamente (466-467). En efecto, en algunos casos, la razón procede de esa forma, pero no en todos, especialmente en la ética. La ética deductiva o racionalista no responde del todo a la naturaleza de lo estudiado, a saber, la moral, pues esta última no es susceptible de una demostración de tal tipo, dado que trata con lo contingente; sólo lo que es necesario puede ser tratado geométricamente. La única necesidad de la que podría hablarse en ética es de la necesidad moral, que ya hemos referido y que, por cierto, es asimismo contingente, aunque fincada en la objetividad.

Como la razón, para él, es incapaz de influir en las acciones, no le queda más remedio que dejar el asunto en el ámbito emotivo o sentimental. Así se aprecia tanto en los casos de parricidio e incesto trabajados por él, en donde no encuentra incluso la diferencia entre el ámbito humano y el animal, y considera que en ambos se daría la misma acción. No habría distinción entre la acción de uno y otro: "Los animales son susceptibles de tener las mismas relaciones entre sí que la especie humana, y, por consiguiente, deberían ser también susceptibles de la misma moralidad en el caso de que la esencia moral consistiera en estas relaciones" (Hume, 1998, p. 468). Por supuesto que si el entendimiento y, con él, la razón, consisten sólo en lo que Hume les atribuye, la conclusión no es más que necesaria. Pero, como hemos reiterado ya, se trata de un error que se encuentra en la propia concepción humeana de entendimiento y razón, por lo cual sus conclusiones no pueden ser sino absurdas: no son las mismas relaciones las que se establecen entre seres humanos y las que los animales establecen entre ellos. La moralidad es un ámbito genuinamente humano y no es compartido con los animales debido a que las potencias intelectivas y volitivas del hombre son distintas a las de los brutos. Por esta causa, Hume plantea el emotivismo como alternativa, y lo hace recurriendo a un ejemplo: el asesinato intencional. Veamos lo que él dice:

Examinadlo desde todos los puntos de vista posibles, a ver si podéis encontrar
esa cuestión de hecho o existencia a que llamáis vicio. Desde cualquier
punto que lo miréis, lo único que encontraréis serán ciertas pasiones,
motivos, voliciones y pensamientos. No existe ninguna otra cuestión de
hecho incluida en esta acción. Mientras os dediquéis a considerar el objeto,
el vicio se os escapará completamente. Nunca podréis descubrirlo hasta el
momento en que dirijáis la reflexión a vuestro propio pecho y encontréis
allí un sentimiento de desaprobación que en vosotros se levanta contra esa
acción. He aquí una cuestión de hecho: pero es objeto del sentimiento, no
de la razón. Está en vosotros mismos, no en el objeto (1998, p. 469).

Hume coloca como principio de la moral a los sentimientos fundamentales, los cuales no son necesariamente sentimientos morales. Los sentimientos de aprobación y desaprobación son una manera de explicar los sentimientos fundamentales de placer y dolor, elevados arquitectónicamente, esto es, como 
principios o fundamentos primeros. Es lo mismo que Gilles Deleuze explica con precisión: "Pero que el placer sea un bien y el dolor un mal, que tendamos al placer y rehuyamos el dolor: he aquí lo que no se halla contenido ni en el dolor ni en el placer; ésa es la operación de los principios" (1996, p. 138). La moral, en el espíritu de la filosofía de Hume, es más sentida que juzgada; siendo así, la virtud es algo agradable y el vicio algo desagradable. Es el sentimiento mismo aquello que causa que el sujeto considere algo (una acción, un carácter, etcétera) como bueno o malo, virtuoso o vicioso, alabable o censurable. Es algo análogo a lo que sucede con los juicios relativos a los gustos, a saber, que la aprobación se halla implicada en el placer inmediato que proporciona aquello que gusta (Hume, 1998, pp. 470-471). De ahí su conclusión: “La virtud se distingue por el placer, y el vicio, por el dolor, que cualquier acción, sentimiento o carácter nos proporciona con sólo verlo y contemplarlo" (475). Sin embargo, aunque las notas expuestas hasta aquí otorgan un panorama suficiente sobre la filosofía moral de Hume, hay que destacar, por otra parte, que el filósofo escocés da una pauta para escapar de su sistema. Se trata de una pauta que él no desarrolla, pero que sí deja apuntada, y que es conocida en la filosofía anglosajona como la teoría del impartial spectator, trabajada más que nada por Adam Smith en The Theory of Moral Sentiments. Hume escribe: "Sólo cuando un carácter es considerado en general y sin referencia a nuestro interés particular causa, esa sensación o sentimiento en virtud del cual lo denominamos moralmente bueno o malo" (472). En este punto, Hume da con tino en lo que suele denominarse "sentimiento moral". Pero, si se hace caso a su teoría en general, no tiene cabida esta tesis, pues el sentimiento no se da en abstracto, sino en el individuo particular, el cual es incapaz sin más de encontrar el punto de vista imparcial que resulta necesario para elevarse del plano individual al general o universal. Sólo en el plano general o imparcial podrá encontrarse un fundamento más sólido para la moral, pues sólo en él es posible construir generalizaciones y no meras individualizaciones, como se desprende de la filosofía moral humeana.

Para abonar más en la discusión con el emotivismo, Adam Smith afirma que luego de las tesis de Hobbes, que propone una doctrina moral basada en el egoísmo, se da una fuerte reacción y se propugna a la razón como principio de la aprobación moral. La razón, anterior a toda legislación o institución positiva, es capaz naturalmente de distinguir las cualidades de lo bueno y lo malo, de lo virtuoso y lo vicioso. Es la razón la que se encarga de indicar la diferencia entre el bien y el mal. Tal es la tesis central, a juicio de Smith, de quienes colocan a la razón como principio de la moral. Ahora bien, su crítica es la siguiente:

Es completamente absurdo e ininteligible suponer que las percepciones primarias de lo bueno y lo malo procedan de la razón, hasta en aquellos casos particulares de cuya experiencia se sacan las reglas generales. Estas percepciones primarias, así como toda experiencia en que cualquier regla general se funda, no pueden ser objeto de la razón, sino de un inmediato 
sentido y emoción. La manera como se forman las reglas generales éticas, es descubriendo que en una gran variedad de casos un modo de conducta constantemente nos agrada de cierta manera, y que, de otro modo, con igual constancia, nos resulta desagradable. Empero, la razón no puede hacer que un objeto resulte por sí mismo agradable o desagradable; la razón sólo puede revelar que tal objeto es medio para obtener algo que sea placentero o no, y de este modo puede hacer que el objeto, por consideración a esa otra cosa, nos resulte agradable o desagradable. Mas nada puede ser agradable o desagradable por sí mismo, que no sea porque así nos lo presenta de inmediato un sentido y sensación. Por lo tanto, si en todos los casos particulares necesariamente nos agrada la virtud por ella misma, y si del mismo modo el vicio nos causa aversión, no puede ser la razón, sino un inmediato sentido y sensación, lo que así nos reconcilie con la una y nos extraña del otro (Smith, 2004, p. 2).

La tesis central de Smith, como puede verse con claridad, es que las normas éticas se forman por referencia al agrado o desagrado constante de las conductas que pueden calificarse moralmente. En resumen, la norma de moralidad responde al agrado o desagrado que nos producen las acciones morales. La razón no es capaz de hacer que un objeto, en este caso un acto moral, sea agradable o desagradable; luego, la razón no es fundamento de la norma moral. Pero debido a que un objeto no es agradable o desagradable per se, pues es evidente que debe serlo para alguna facultad, es necesario postular una que sea capaz de discernir entre una y otra ponderación. Smith, en consecuencia, asegura que lo que nos agrada es virtuoso y lo que nos desagrada es vicioso; y aquello que discierne entre una y otra ponderación es "un inmediato sentido y sensación". ¿Qué es, pues, esta facultad, que ha apuntado Smith, que debe tener por objeto lo particular y no lo general? Se trata de un "sentido inmediato y una emoción". Lo que distingue lo agradable $y$, en consecuencia, lo virtuoso, de lo desagradable $y$, por tanto, lo vicioso, es esa facultad anotada. Pero Smith se separa de Hutcheson, quien es el primero en proponer un sentido de esta naturaleza, como intermedio entre la pura sensación y la razón, a saber, the moral sense. Smith se inclina más bien por un poder de percepción que no es nuevo, como el de Hutcheson, sino que está implicado en los otros. Este poder es la "simpatía", "de la que siempre se ha tenido debida cuenta y de la que la mente está manifiestamente dotada, es, piensan, suficiente para explicar todos los afectos atribuidos a aquella facultad especial" (Smith, 2004, p. 2) ${ }^{1}$.

Ahora bien, ¿es la simpatía una facultad? ¿O es el acto de una facultad? Sobre estas preguntas es preciso reflexionar. Si la simpatía fuera una facultad, bastaría con esperar que naturalmente se desarrolle; de ser así, y en consecuencia, la moralidad tendría que esperar a que se desarrolle de alguna manera la "facultad

\footnotetext{
'Sobre las distintas formas de simpatía, a saber, la simpatía como contagio, la simpatía como identificación, la simpatía mutua y la simpatía mutua moral, además de una explicación suficiente sobre el espectador imparcial, Cf. Carrasco, 2009, pp. 81-96.
} 
de la simpatía". Pero, ¿qué sucede si se atrofia? Además, ¿qué tipo de facultad es? ¿Se trata de una facultad dedicada, como exigiría la cláusula, a lo particular? ¿Cómo es que la noción de obligación puede darse en una facultad de este cuño? Nos parece que elevar a facultad o potencia a la simpatía sería desvirtuarla. Más bien parece ser un acto, y es uno justamente en donde confluyen varias potencias, desde las sensitivas hasta las intelectuales. Para obtener el acto de la simpatía, es necesario que un sujeto sea afectado por un objeto y su circunstancia, pues puede haber simpatía tanto por una persona, como por una acción o una circunstancia. Así pues, es necesario aceptar un objeto y un sujeto; el objeto es simpático para un sujeto, que encuentra en aquél elementos para identificarse, pues la simpatía es, como revela su nombre, padecer-con, en este caso, con un objeto. Ahora bien, al revelarse que se trata de un padecimiento conjunto, es necesario aceptar que implica el concurso de una facultad capaz de percibir lo singular y no sólo lo universal; de ahí que Smith, nos parece, insista en su estado intermedio entre la pura sensibilidad y la razón. Pero, si fuera el caso, y la razón no tuviera concurso en el acto, entonces podría afirmarse que los brutos poseen esta facultad caracterizada como simpática. Lo cual, por otra parte, parece ser cierto. Luego, en los animales también se daría la moralidad, pues son capaces de compadecer a un objeto. Empero, esta conclusión se nos presenta absurda, por lo cual es necesario recurrir a una facultad exclusivamente humana para dar cuenta de la simpatía, y ésta parece ser precisamente la razón. La razón, a nuestro juicio, es capaz de motivar la simpatía por un objeto, o bien el desagrado hacia éste. Y lo hace a través de una facultad de índole sensitiva, a la que llamamos imaginación ${ }^{2}$. Es a través de esta facultad que el animal es susceptible de simpatizar con el objeto, pero en el hombre la imaginación es motivada por la inteligencia, que encuentra precisamente razones suficientes para simpatizar con el objeto. Esto pone de relieve que consideramos, por nuestra parte, que las facultades humanas se dividen en cognoscitivas (sensibles y el intelecto) y apetitivas (apetito sensitivo y apetito racional). Todo sentimiento parece ser un acto o estado del apetito, el cual va unido a algún tipo de conocimiento, sea éste obscuro (es decir, sin motivo aparente) o sensitivo (una suerte de intuición en este plano).

Parece que para Smith basta con que haya sentimientos de aprobación hacia un objeto para que este último pueda considerarse bueno y virtuoso. Empero, vemos que su postura resulta insuficiente por varias razones. El principal argumento es que hay una confusión entre las tendencias (que pueden ser benévolas o sociales) y el deber moral. Una cosa es la materia de la moralidad, que en este caso puede

${ }^{2}$ El propio Smith tiene presente que se trata de la imaginación, o que al menos ella concursa en el acto, pues asegura que "sólo por medio de la imaginación nos es posible concebir cuáles sean sus sensaciones [las de un semejante]. Ni, tampoco, puede esta facultad auxiliarnos en ese sentido de otro modo que no sea representándonos las propias sensaciones si nos encontrásemos en su lugar. Nuestra imaginación tan sólo reproduce las impresiones de nuestros propios sentidos, no las ajenas. Por medio de la imaginación, nos ponemos en el lugar del otro" (Smith, 2004, p. 30). 
aplicarse a las tendencias, y otra la forma de la moralidad, que es el cuño que le imprime la inteligencia a ciertas tendencias. En efecto, una cosa es la tendencia y otra el deber que constata (y juzga) la inteligencia humana. La tendencia, por sí misma, es sólo materia de moralidad, pues no implica por ningún motivo al concepto de obligación moral. Empero, para que algo sea moralmente obligatorio, es necesario que la inteligencia juzgue que la materia es digna en algún sentido (en este caso moral). La tendencia, por sí sola, no es formalmente obligatoria; se constituye en obligatoria cuando la inteligencia juzga que la tendencia es digna de seguirse.

Siendo así, postular una facultad como lo ha hecho la escuela de Hutcheson nos parece gratuito, pues en último análisis requiere de una facultad que juzgue que la simpatía que se tiene por un objeto es algo digno de ser estimado así. En terminología más actual, la simpatía parece ser más bien un movimiento del apetito que se siente identificado por un objeto, Ilegando incluso a apetecerlo en cuanto tal; pero para que sea un apetito moralmente bueno, se requiere del concurso de la razón, que estima si tal objeto es digno justamente de apetecerse; esta estimación es, a nuestro parecer, la valoración. En consecuencia, la simpatía requiere de la aprobación de la razón para ser moralmente virtuosa.

Finalmente, y para concluir este punto, Ferber brinda dos argumentos plausibles contra el emotivismo, en donde englobamos tanto a Hume como a Smith, que nos parece importante tener presentes. En primer lugar, esta postura va contra "la exigencia de generalización que asociamos al concepto de tales proposiciones básicas morales"; en segundo lugar, "las simples expresiones o relatos sobre los propios sentimientos pueden diferir notablemente acerca de los mismos hechos" (Ferber, 1995, pp. 160-161). Tomemos los argumentos como conductores, aunque sin seguir fielmente a Ferber. El primer argumento puede reelaborarse de esta manera: si el sentimiento moral es la guía del actuar moral, y este sentimiento, como su mismo nombre lo indica, está fincado en el movimiento producido por las propias sensaciones, luego es imposible hacer generalizaciones; en consecuencia, la exigencia de la teoría moral de hacer generalizaciones, esto es, de efectuar juicios o fórmulas morales con un carácter general, sería imposible si el criterio es sentimental; en consecuencia, el sentimiento moral es insuficiente para dar cuenta de la moralidad. El segundo argumento puede reelaborarse así: si el sentimiento moral depende del estado de ánimo de quien observa un hecho, y el hecho es posible que sea deformado por aquél; luego no puede representar un suelo seguro para elaborar una teoría moral. Siendo así, veamos otra postura que brinda, a juicio nuestro, mejores argumentos, a saber, el institucionalismo. 


\section{El institucionalismo: hacia un institucionalismo humano}

El institucionalismo intenta colocarse a medio camino entre el cognitivismo y el emotivismo. John Searle, con su Speech Acts, es considerado paradigma de esta postura. Así lo señala el propio Ferber, aunque será conveniente revisar qué dice precisamente Searle en su libro mencionado. Ahí, el filósofo norteamericano habla de los hechos brutos (brute facts) del mundo, cuyo conocimiento es generalmente considerado en términos de simples observaciones empíricas que registran experiencias sensitivas. Pero él mismo cae en la cuenta de que buena parte del lenguaje no consta de conceptos que sean parte de la representación o conocimiento antedicho, esto es, del conocimiento empírico de los hechos brutos:

Notoriamente, los enunciados de la ética y la estética no son asimilables con facilidad a esta representación, y los filósofos que pretenden aceptarlas dicen que no son enunciados en absoluto, sino solamente expresiones de emociones, o bien que son meramente enunciados autobiográficos de tipo psicológico que dan cuenta, como decía Hume, de sentimientos (Searle, 1969, p. 50)

Entonces, iqueda dejar a los enunciados éticos (y estéticos) como algo meramente pasional? ¿No tienen ninguna relación con el mundo de los hechos? La respuesta que apunta Searle es negativa, pues postula un nuevo género de hechos, a saber, los institutional facts. Retomemos el ejemplo del football americano que elabora el propio Searle. Un observador externo puede describir un juego de football americano efectuando enunciados que engarzan con los hechos brutos, por ejemplo, cada determinado tiempo los individuos de un mismo color se agrupan de cierta manera, se dirigen hacia la misma zona del campo, etcétera. Pero, aún con todo, el observador no logra describir el football americano.

¿Qué falta a su descripción? Lo que falta son aquellos conceptos que están respaldados por reglas constitutivas, conceptos como touchdown, fuera de juego, partido, puntos, tiempo fuera, etcetera, y consecuentemente lo que les falta son todos los verdaderos enunciados que pueden hacerse sobre un juego de football usando tales conceptos. Los enunciados faltantes son precisamente lo que describe el fenómeno que se desarrolla como un juego de football (Searle, 1969, p. 52) .

\footnotetext{
El texto original: "Notoriously, statements in ethics and esthetics are not readily assimilable to this picture, and philosophers who have accepted the picture have tended to deal with them either by saying that they were not statements at all but mere expressions of emotions, or that such statements were simply autobiographical statements of a psychological kind, recording, as Hume says, sentiments".

${ }^{4}$ El texto original: "What is missing from their description? What is missing are those concepts which are backed by constitutive rules, concepts such as touchdown, offside, game, points, first down, time out, etc., and consequently what is missing are all the true statements one can make about a football game using those concepts. The missing statements are precisely what describes the phenomenon on the field as a game of football".
} 
En efecto, hay enunciados que no engarzan precisamente con los hechos brutos, aunque ello no implica necesariamente estar desvinculados de ellos. Pero antes de sostener esto último, retomemos nuevamente a Searle, quien desarrolla su teoría del siguiente modo. Asegura, primero, que se trata de hechos, pero son hechos que presuponen la existencia de ciertas instituciones humanas. En segundo lugar, asienta que estas instituciones son sistemas constituidos por reglas. En tercer lugar, propone que todo sistema de reglas tiene la siguiente forma: " $X$ cuenta como $Y$ en el contexto $C^{\prime \prime}$ (Searle, 1969, pp. 51-52). Formalmente, no parecen encontrarse huecos en la teoría de Searle. Pero no sucede lo mismo si se aplica, como hace Ferber, a los hechos morales. Dice este último:

Los hechos morales no son hechos que se sustenten en sí mismos, ya sea en el mundo físico o en el mundo invisible y metafísico, según demuestra el cognitivismo, como tampoco son simples hechos psíquicos subjetivos, tal como propone el emotivismo descriptivo. Tampoco se trata por todo ello de hechos inexistentes, sino que existen ciertamente hechos morales, aunque de índole institucional. En consecuencia, la moral no es algo puramente objetivo ni algo simplemente subjetivo, sino esencialmente algo social, a saber: una institución creada por los hombres (1995, p. 162).

Como se aprecia, Ferber propone que los hechos morales existen, y que se trata de institutional facts, a la usanza de Searle. Los hechos morales son hechos esencialmente sociales. Hasta aquí puede concederse por el momento lo que asegura Ferber. Pero no parece encontrarse alguna premisa intermedia entre el enunciado "la moral es algo esencialmente social" y "la moral es una institución creada por los hombres". Ha pasado Ferber de un enunciado que señala un fenómeno social a una creación humana. Presupone que todo lo que es social es creación humana. Pero esta presuposición no es falsa; sucede que se contrapone a otro hecho: el hombre es esencialmente moral, aunque la manera en que construye la moral puede considerarse "creativo" en este contexto. ¿Cómo conjuntar el fenómeno esencialmente humano de ser un ente moral y la creación, pareciera que ex nihilo, de la moralidad? Aquí se halla, a nuestro juicio, el quid de este asunto.

Parece que Ferber confunde, en el fondo, el valor con la valoración, al igual que sucede con el emotivismo, y éste sería el mismo error del institucionalismo moral frente a una postura cognitivista o, mejor, objetivista moral. Nuestra interpretación tiene fundamento si se tiene presente la siguiente tesis del filósofo alemán:

Sentimos horror ante un asesinato premeditado porque así lo hemos aprendido de nuestros padres, profesores y compañeros, y también los demás sienten lo mismo. De habernos socializado hace tres mil años, o tal vez sólo trescientos, ciertamente que la mayor parte de nosotros habríamos sentido horror e irritación por el asesinato de un miembro de la propia familia, tribu o pueblo, pero habríamos experimentado quizá indiferencia y hasta satisfacción por la eliminación del miembro de otra familia, de otra tribu o de otro pueblo (Ferber, 1995, p. 162). 
No parece ser falsa la apreciación de Ferber, pero se trata de valoraciones contingentes. En efecto, la valoración, esto es, el juicio práctico, es de facto variable. Pero de ahí no se sigue que el juicio práctico siempre sea correcto, que en este caso consiste en ser un juicio recto que eventualmente conduce a un acto externo bueno.

De acuerdo con la visión del filósofo alemán, los hechos morales son hechos institucionales, es decir, establecidos por los seres humanos. Pero, ipor qué no establecer que se trata de hechos que son naturales en el hombre? ¿No es más conveniente y hasta certero que el hombre, por el hecho de ser tal, pueda ser capaz de descubrir algunos hechos morales que sean absolutamente reprobables? No quiere decir que de facto se valore siempre de acuerdo con estos patrones, sino simplemente de fundamentar que hay hechos que, de acuerdo con la propia naturaleza humana, son absolutamente rechazables desde el punto de vista moral. Tal es el caso, por ejemplo, del genocidio. Porque, de ser pura y simplemente institucionales, hechos morales como el genocidio podrían llegar a aceptarse como moralmente buenos si la comunidad (institución) así lo estima. ¿No será preferible sostener que la "institución humana", a la cual pertenece todo hombre posible, ha de valorar como reprobables ciertos hechos morales, siempre y cuando se atenga a los valores propios de las cosas en cuestión? Tal es precisamente el fenómeno moral: no se trata de convencer al agente en todos los casos, sino que éste debe someterse en muchos de ellos a la norma moral, ante la cual permanece libre. Pero alguno preguntará: ¿dónde está la norma moral? La norma moral próxima está en el ser mismo de las cosas que conoce el hombre; este conocimiento directo de las cosas es aquello que funge como regla o norma para la valoración moral del hombre. Así, aunque las distintas instituciones del hombre valoren de facto un objeto o hecho de cierta manera, no se sigue que efectivamente se sometan a la norma moral; es más, la norma moral es la que ha de servir como guía para revisar si la valoración es verdadera; y esta guía para revisar la valoración es el valor mismo de las cosas, que se funda en el ser de cada una de ellas.

Si se admite que ciertos actos sólo tienen sentido al interior de la institución en la cual se efectúan, sólo se posterga el problema, pues la pregunta ¿es bueno, por ejemplo, el genocidio?, obtendrá su respuesta de acuerdo con la institución en la cual se formule. Searle y Ferber tienen razón en que ciertos enunciados tienen sentido al interior de ciertas instituciones. Pero si se considera que la institución en el caso moral equivale al mismo hombre, entonces la moralidad tiene sentido mientras el hombre exista, y no en tanto que crea o no instituciones, pues la moralidad no se instituye de la misma manera en que se instituye un juego, esto es, a través de reglas completamente arbitrarias. Por el contrario, la moralidad es co-extensiva con el hombre mismo, de tal suerte que, aunque las morales históricas sean una construcción y, por tanto, una institución, no lo son absolutamente, pues la moralidad es esencial al hombre. 
En este sentido, no toda moral puede estar sometida a las convenciones o reglas arbitrarias. Las reglas morales más básicas no se encuentran sometidas al arbitrio humano, sino sólo sus derivaciones, que se efectúan una vez interpretadas las distintas variables que entran en juego. Además, la convención implica que se acuerda sobre algo, y este algo es ya objetivo. Por tanto, no todo queda al arbitrio humano. Por ejemplo, el valor de la persona humana tiene su fundamento en las capacidades que, aunque potencialmente, están presentes en el hombre naturalmente. La moralidad ha legislado sobre su valor, pero se trata de algo que está fuera de la convención.

\section{La importancia de la voluntad desde el cognitivismo u objetivismo moral}

Varias posturas morales parecen no tener muy en claro una antropología filosófica que les sirva de sustento para construir sus consecuentes edificios éticos. Y es que, nos parece, la ética es consecuencia de una antropología filosófica, y esta última, a su vez, de una ontología conectada con una metafísica. De una u otra forma, toda ética tiene como base una ontología, sea ésta naturalista, institucionalista y hasta emotivista. Nos parece que las carencias que hemos señalado en este trabajo que tienen que ver con la ética, poseen su base en una antropología filosófica. Es cierto que, por ejemplo, en el emotivismo la antropología filosófica es muy trabajada, ya desde los tiempos de Locke y Hume, y que ambos han construido antropologías filosóficas. Empero, y como hemos puesto de manifiesto aunque sea someramente, pues hacerlo exhaustivamente exigiría un trabajo más amplio, varias de sus posturas antropológicas son criticables, especialmente en el papel que brindan a la razón. Algo semejante apreciamos en el institucionalismo, pues tal parece que reduce al hombre a un mero agregado social, incapaz de salir de las instituciones que él mismo ha creado, lo cual implica, en el campo moral, que sería imposible salir de los cánones impuestos por la comunidad social a la que se pertenece. Pero esto último es contrario a la experiencia: tanto individual como socialmente, parece que el cambio social es una constante; si no fuera así, un individuo permanecería inalterado a lo largo de su existencia, así como las distintas instituciones humanas.

El cognitivismo, para completar la acertada tesis de que es a partir del conocimiento de lo que son las cosas que podemos tener un suelo moral firme, requiere recurrir al elemento valorativo que es motivado por una facultad especial: la voluntad. Sin la voluntad, el fenómeno moral aparecería ininteligible, pues no habría una facultad capaz de ser digna de imputación, en este caso, imputación moral. En este sentido, Bocheński deja anotado algo que podría resultar crucial para comprender al valor y la consecuente, aunque aleatoria, valoración: "Sucede, efectivamente, en los valores que la visión no depende sólo de la inteligencia, sino, sobre todo, de la voluntad" (1992, p. 77). La captación del valor, tal como 
lo han señalado los más preclaros axiólogos del siglo XX, es afectiva. Pero quien capta el valor es el entendimiento, el cual se ve afectado volitivamente hablando. Ahí tiene su origen el sentimiento moral, pues es la manera afectiva que viene acompañando a la captación del valor.

La filosofía de Antonio Rosmini parece poner orden a esta serie de problemas. Retomemos algunas directrices generales del pensamiento del filósofo roveretano para nuestros propósitos (Rosmini, 2010) ${ }^{5}$. En primer lugar, ¿cómo afirmar que la voluntad entra efectivamente en la valoración? Básicamente concibiendo correctamente a la voluntad, que no es, por mucho, irracional (como se desprendería de Schopenhauer, por ejemplo), esto es, un apetito que no tiene que ver con la racionalidad, sino que incluso la maldad de la cual ella es origen es más que razonada, aunque irracional en cuanto niega lo que le brinda la razón, entendiendo por razón lo que el propio entendimiento nos muestra como el ser de las cosas. Sólo así la voluntad es irracional. Pero, in nuce, la voluntad apetece siempre racionalmente, pues es capaz de dar cuenta de por qué actúa decide o apetece más una cosa que otra. Nos parece que así es que puede comprenderse por qué la voluntad es fundamental al momento de la valoración: la voluntad es capaz de motivar el juicio práctico sobre aquello que conoce intelectualmente $y$, así, es que entra en la valoración. El tomismo también ha señalado reiteradamente que la voluntad, aunque dependiente del entendimiento, parece tener la última palabra al momento de la valoración, es decir, al momento de apreciar una cosa inteligible desde el punto de vista de su bondad, no en cuanto a su constitución per se, sino en cuanto a su reconocimiento.

Teniendo como trasfondo una caracterización como la anterior en torno a la voluntad, la razón, el valor y la valoración, es posible concluir lo siguiente: en primer lugar, en el acto moral entran en juego no sólo las capacidades sensitivas del hombre, sino, y sobre todo como forma de la moralidad, el entendimiento y la voluntad. El entendimiento, aunque lo hemos presupuesto en este trabajo, es capaz de conocer el ser de las cosas. De no aceptarse esta tesis, la moralidad se tornaría ininteligible, pues si el hombre es incapaz de conocer lo que son las cosas, ¿̇ónde encontraría la sede para fundamentar que un acto es bueno o malo moralmente? Quedaría como única vía el subjetivismo, que nos lleva, como vimos, a una moral de corte emotivista. A lo sumo, podría llevarnos a un institucionalismo.

En segundo lugar, la distinción anterior nos lleva a precisar que el valor y la valoración son dos cosas distintas, como pide Bocheński. En efecto, el valor es captado por la propia inteligencia o razón, mientras que la valoración es un juicio práctico motivado por la voluntad, que es la potencia para adherirse al ser: fundamentalmente lo hace amándolo. Si la voluntad ama al ser en cuanto ama lo

${ }^{5}$ Tenemos presentes todas las obras morales del filósofo italiano, pero en especial: Principios de la ciencia moral. 
que es él, entonces parece conducirse moralmente bien; si la voluntad ama al ser no en cuanto es en sí mismo, sino como ella quiere, entonces parece conducirse inmoralmente. La moralidad, la esencia de la moralidad, se encuentra claramente en esta postura, pues la exigencia moral no es otra cosa que la exigencia que cada ente tiene de ser reconocido por aquello que es, y es la voluntad la potencia o instancia humana que tiene este poder, el cual, al ejercerlo, lo lleva a perfeccionarse o no en cuanto tal, es decir, moralmente. Los otros sistemas, como son el emotivismo y el institucionalismo, no logran hallar la esencia de la moralidad, sino que permanecen en la confusión antropológica que los lleva a teorías éticas subjetivistas. El cognitivismo u objetivismo moral, por su parte, si quiere ser completo, requiere tener presente siempre y en todo momento, para dar cuenta de la moralidad, de la potencia que hemos denominado, junto a la tradición, voluntad. Entendimiento o razón y voluntad, así como la gama de sensaciones y afecciones no sólo racionales, sino también animales, es preciso tenerlas presentes para comprender el fenómeno moral y, en consecuencia, el valor y la valoración morales.

\section{Referencias}

Bocheński, J. M. (1992). Introducción al pensamiento filosófico. Barcelona: Herder.

Carrasco, M. (2009). De Hutcheson a Smith: un sentimentalismo sofisticado. Revista de filosofía, 65, 81-96.

Deleuze, G. (1996). Empirismo y subjetividad. Barcelona: Gedisa.

Ferber, R. (1995). Conceptos fundamentales de la filosofía. Barcelona: Herder.

Hume, D. (1998). Tratado de la naturaleza humana. Madrid: Tecnos.

Rosmini, A. (2010). Principios de la ciencia moral. México: Universidad Veracruzana y Plaza y Valdés.

Searle, J. (1969). Speech acts. An Essay in the Philosophy of Language. Cambridge: Cambridge University Press.

Smith, A. (2004). Teoría de los sentimientos morales. México: Fondo de Cultura Económica. 